\title{
Necessity to Invest in Cost-Effective Interventions for the Prevention of Hearing Loss
}

\section{Shrivastava SR*, Shrivastava PS and Ramasamy J}

Department of Community Medicine, Shri Sathya Sai Medical College and Research Institute, Tamil Nadu, India

\section{Introduction}

Globally, millions of people are living with hearing loss and even lack access to desired ear and hearing care provisions, of which majority are from low and middle income nations [1]. Even though, the answers are available in most of the dimensions of hearing loss (viz. aetiology, preventive measures, early detection tools, therapeutic interventions, etc.), most of them are deprived of adequate care and thus are having limited opportunities for wellbeing [2]. In-fact, the findings of a recent global estimate suggest that almost 360 million people are suffering from disabling hearing loss, while more than 325 million people have chronic ear infections [1]. Furthermore, close to $33 \%$ of elderly people are having hearing loss and in excess of a billion people are at the potential risk of developing hearing loss owing to listening to music at loud volumes and for a prolonged time interval [1,2].

There is no doubt that hearing loss has been associated with massive impacts on communication, educational or vocational opportunities, and social and emotional disturbances [1]. It is an alarming fact that almost all the middle and low income nations have no strategies to address the concern of hearing loss and is predominantly due to the limited availability of resources [1-3]. Moreover, any evidence pertaining to the involved economic costs can be a powerful tool for policy-makers in planning their health care related budgets [3].

In order to prioritize the issue of hearing loss among policy makers, the cost of hearing loss has been estimated by the World Health Organization (WHO) and its partners [1]. It has been estimated that the mean cost of moderate or higher degree of hearing loss to the healthcare sector for adults and children is $\$ 87$ billion (excluding the cost of providing hearing devices) and education sector for children aged 5-14 years is $\$ 3.9$ billion. In addition, more than $\$ 100$ billion cost has been incurred each year due to the loss of income/job or early retirement of people with hearing loss [3]. Further, billions of dollars are spent on societal costs also each year [3]. Thus, the total mean annual cost of unaddressed hearing loss is $\$ 770$ billion worldwide [3].
It is extremely important to understand that most of the available interventions to address hearing loss are cost-effective, whether it relates to its prevention (like early detection and management of infections, prevention of noise-induced hearing loss, strengthening of maternal and child welfare strategies, training of health professionals in ear and hearing care, creating awareness about hearing health among different stakeholders, including the general population, etc.), screening activities among different age-groups or even use of hearing aids by the patients $[1,3]$.

The WHO emphasizes the need for the global stakeholders to explore the cost incurred due to unattended hearing loss and to motivate them to implement cost-effective interventions [3]. This essentially calls for the rational allocation of resources, integration of the ear and hearing care with the health systems, capacity building, implementation of early identification and intervention programs, and to increase awareness among all sections of the community [1-3]. Moreover, it is quite possible that those who develop hearing loss can regain their complete potential through early detection, rehabilitation, use of hearing devices, education and through their empowerment $[1,3]$.

\section{Conclusion}

To conclude, it is vital to note that unaddressed hearing loss accounts for substantial financial burden on the healthcare system and the economy as a whole. It is the need of the hour to implement costeffective interventions for the prevention and control of hearing loss.

\section{References}

1. World Health Organization (2017) Action for hearing loss: Make a sound investment.

2. Shrivastava SR, Shrivastava PS, Ramasamy J (2016) Supporting the global initiative of preventing childhood hearing loss: Act now, here's how. Noise Health 18: 280-281.

3. World Health Organization (2017) Global costs of unaddressed hearing loss and cost-effectiveness of interventions - Executive Summary. Geneva: WHO press, pp: $1-4$
*Corresponding author: Shrivastava SR, Department of Community Medicine, Shri Sathya Sai Medical College and Research Institute, Tamil Nadu, India, Tel: +919884227224; E-mail: drshrishri2008@gmail.com

Received July 28, 2017; Accepted July 29, 2017; Published August 05, 2017

Citation: Shrivastava SR, Shrivastava PS, Ramasamy J (2017) Necessity to Invest in Cost-Effective Interventions for the Prevention of Hearing Loss. Prim Health Care 7: e121. doi: 10.4172/2167-1079.1000e121

Copyright: $\odot 2017$ Shrivastava SR, et al. This is an open-access article distributed under the terms of the Creative Commons Attribution License, which permits unrestricted use, distribution, and reproduction in any medium, provided the original author and source are credited. 